\title{
Doxycycline Induced Extensive Esophageal Ulcerations: Case Report and Review of the Literature
}

\author{
Elsayed Saad Abd Elbaser \\ Tropical Medicine Department, Faculty of Medicine, Zagazig University, Egypt \\ Email: dr.sayedsaad79@gmail.com
}

\begin{abstract}
Drug induced esophageal disease is common. Doxycycline is one of the commonest causes of drug induced esophageal disease. The diagnosis is usually clinical but endoscopy is the gold standard diagnostic modality. Treatment is mainly depending on discontinuation of the offending medication. If left untreated it can have serious consequences like delayed esophageal stricture. A 22-year-old male had been prescribed doxycycline capsules for acne and developed odynophagia. Endoscopy revealed extensive esophageal ulcerations. He was managed symptomatically with proton pump inhibitors and his odynophagia improved over a period of five days. He was discharged with proper advice regarding medication ingestion and proton pump inhibitor for four weeks.
\end{abstract}

\section{INTRODUCTION}

Drug induced esophageal disease (DIOD) was first described in a patient who ingested potassium chloride tablets [1]. Since this initial report, the frequency of pill-induced esophageal injury has continued to grow and more than 1000 cases of drug induced esophageal injury have been reported [2]. The incidence of pill esophagitis has been estimated as approximately four cases per 100,000 per year [3]. Drug induced esophageal diseases are usually under estimated, as many cases usually resolve rapidly after cessation of the medication [4].

Doxycycline is one of the commonest causes of drug induced oesophageal ulcers, it is commonly prescribed for pelvic inflammatory disease and acne, hence doxycycline induced oesophageal injury is more common in females [5].

\section{Pathogenesis}

The mechanism of DIOD is believed to be due to prolonged contact of the caustic contents of the medication with the esophageal mucosa [6]. The typical esophageal lesion showing a small punched out ulcer in the area that was in contact with a high concentration of medication. The site of injury is frequently found in areas in which the esophageal lumen is compromised by the aortic arch, the esophago-gastric junction, or an enlarged left atrium. Thus, medication-induced injury requires that the pill or capsule remain in the esophagus for a prolonged interval, and that its contents be caustic to the esophageal mucosa [5].

The situations that enhance pill retention, thereby increasing the likelihood of esophageal injury may include: lack of an adequate fluid intake and ingestion of a pill immediately prior to sleep, since salivation and swallowing frequency are markedly reduced during sleep [7].

Patients with cardiac disease, particularly following thoracotomy, also appear to be at increased risk. Although this may be due to anatomical changes in the mediastinum, these patients are often more elderly and likely to be taking commonly implicated drugs, such as quinidine or potassium compounds. In contrast, pill-induced esophageal injury has not been commonly reported in patients with known esophageal motility disorders. These patients are often more attentive to swallowing function, which may provide protection when pills are ingested [6].

\section{Clinical presentation}

Patients with DIOD will often present with the sudden onset of odynophagia and retrosternal pain with no history of prior esophageal disease; the pain may be so severe that swallowing saliva is difficult. Patients often relate the onset of symptoms to the swallowing of a pill without water, commonly at bedtime. Typical scenarios include the teenage patient with acne who takes tetracycline at bedtime without water, and the elderly patient in a nursing care facility given a number of medications with a small amount of water while recumbent prior to sleep [5].

\section{Diagnosis}

Medication-induced esophagitis is often suspected when typical symptoms appear abruptly after 
improper ingestion of a pill known to cause esophageal injury. Confirmatory endoscopy or barium radiography is more important in patients with particularly sever or atypical symptoms

Upper endoscopy is the most sensitive procedure; findings are abnormal in virtually 100 percent of cases [3]. Endoscopy is also helpful to rule out alternate diagnoses such as reflux esophagitis, infectious esophagitis, or malignancy. The typical endoscopic appearance of pill-induced esophageal injury is a discrete esophageal ulcer with relatively normal surrounding mucosa [1]. However, others reported doxycycline induced both esophageal and gastric ulcerations [8].

Here we present a severe, unusual endoscopic appearance of a case of doxycycline-induced esophageal ulcers at mid-esophageal segment.

\section{CASE REPORT}

The patient was a 22 years old male who had been prescribed doxycycline $100 \mathrm{mg}$ capsule b.i.d. for acne by pharmacist. He took the first dose of doxycycline $100 \mathrm{mg}$ capsule at $4 \mathrm{pm}$ and 2 hours later he develops mild midsternal pain, one hour after the second dose which was taken at 4 am with no water and went to bed immediately,

he wake up with sever retrosternal chest pain and odynophagia. The pain was burning in character and was located midsternal. It was constant in nature with fluctuations in severity. Swallowing solids and liquids significantly aggravated his pain. There was no history of dysphagia, abdominal pain, fever, melena, rectal bleeding or weight loss. His past medical history was irrelevant, he was a nonsmoker and denied using alcohol, aspirin or non-steroidal anti inflammatory drugs (NSAIDs). His physical examination was normal.

Initial investigations revealed a normal complete blood count, normal liver and kidney function tests and a negative troponin. His chest $\mathrm{x}$ ray was normal. An upper endoscopy revealed circumferential extensive ulcerations with irregular, hyperemic mucosa which bleeds easily on touch and covered with whitish fibrin at the level of the mid-esophagus (Figure 1).

According to the previous data he was diagnosed to have doxycycline induced esophageal ulcerations, so doxycyclin was discontinued and proton pump inhibitor was given intravenously. The patient returned after 5 days and experienced improvement of his symptoms and told that he starts oral feeding normally.

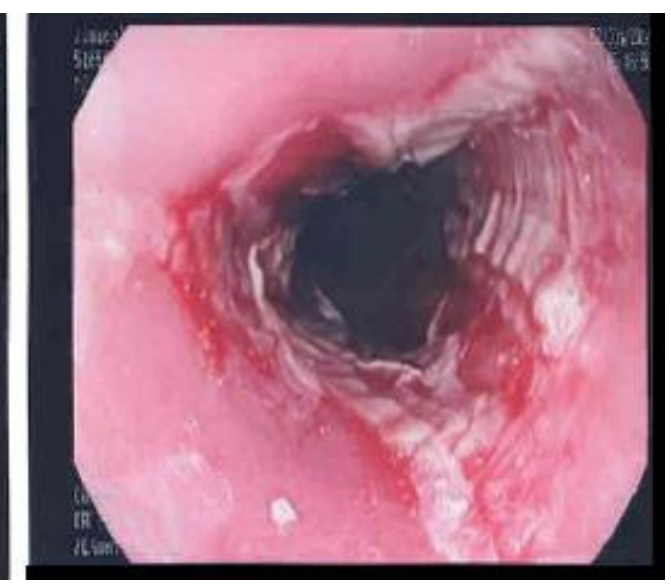

Figure (1): Extensive ulcerations of the mid-esophagus

\section{DISCUSSION}

Drug induced esophageal disease is a common condition that is under reported, it was first reported in the 1970 [9]. Antibiotics account for $50-60 \%$ of drug related esophageal toxicity and tetracyclines, in particular doxycycline, are commonly implicated [10].
Patient factors have been implicated in the causation of oesophageal injury; an elderly patient who takes multiple medications is at high risk for oesophageal injury. Such patients may not remember the advice given by pharmacists. Patient with pre-existing oesophageal diseases such as reflux oesophagitis, scleroderma or oesophageal motility disorders also fall into this high-risk group. 
Our case, demonstrates that even young and healthy individuals are not immune to drug induced injury and there are other factors that are equally important. However, among the reported cases of pill-induced injury, the proportion of the patients having a motility disorder such as achalasia and scleroderma or an anatomical narrowing such as tumor or stricture is low [11].

Medication factors play an important role in drug induced esophageal disease. Medicine taken with less or no water or taken at bed time may remain in contact with the oesophageal mucosa for a long duration and can cause direct mucosal injury [12]. There is decreased salivation and swallowing during sleep and this may increase the transit time through the esophagus. Liquid formulations are less likely to cause oesophageal injury compared to tablets. Sustained release formulations may cause oesophageal injury as they tend to be large in size and hence difficult to swallow. Gelatin based capsules may stick in the oesophageal lumen at the sites of anatomical narrowing (e.g. aortic arch) or pathological narrowing (e.g. enlarged left atrium) and can cause local mucosal injury. The chemical nature of the formulation is also important in the causation of damage. Most of the esophagus damaging medicines are acidic and cause direct toxic effects when they remain in contact with the mucosa for a long duration. Doxycycline can produce a $\mathrm{pH}$ of less than three when dissolved in $10 \mathrm{ml}$ of water or saliva [13]. It is also shown that doxycycline capsules remain in oesophagus for three times long as doxycycline tablets [14].

Our patient was taking doxycycline capsules at bed time with minimal water. In a reported series of eight patients with tetracycline induced oesophagitis, seven patients were taking the drug in capsule form [15]. It is recommended that such medicines be taken in the sitting or standing position and with at least $100 \mathrm{ml}$ of water. It has been reported that a shorter oesophageal transit time occurs when medication is swallowed with more liquids [16]. Also, it is advisable to remain upright for at least 15 minutes after the ingestion of medicine [4].

A detailed history and high index of suspicion is the key to an accurate diagnosis. The common symptoms of drug induced esophageal disease are dysphagia, odynophagia and retrosternal chest pain. In one case series that examined 36 patients with doxycycline related esophageal toxicity, $94 \%$ of patients had odynophagia, $80 \%$ had retrosternal chest pain and 54\% had dysphagia [5]. The symptoms start within hours or days after ingestion of medicine and typically get better within a few days of discontinuing the drug [11].

Endoscopy is the gold standard diagnostic modality. The common endoscopic finding is one to several discrete shallow, small ulcers in the mid-esophagus [5]. Particles of medicine can also be found at the site or ulcer formation. Our patient had circumferential extensive ulcerations with irregular, hyperemic mucosa which bleeds easily on touch and covered with whitish fibrin at the level of the mid-esophagus (Figure 1).

Drug induced esophageal disease is self-limiting and symptoms usually improve on discontinuation of the medicine. Our patient already stopped taking doxycycline after the first day. Proton pump inhibitors or $\mathrm{H} 2$ receptor antagonists have no proven role in the absence of reflux oesophagitis. Topical protective agents and local anaesthetics such as liquid sucralfate or lignocaine may be of benefit for ulcer healing and pain relief. Delayed oesophageal stricture formation may require endoscopic dilatation [3]. The simple advice of swallowing medication with plenty of water in an upright position can prevent the consequence of erosive oesophagitis.

Funding: Non.

Conflicts of interest: The authors declare no conflict of interest.

Ethical approval: Not needed.

\section{REFERENCES}

1. Pemberton J. Oesophageal obstruction and ulceration caused by oral potassium therapy. Br Heart J. Mar 1970; 32(2): 267-268.

2. Cellier C. Drug induced oesophageal mucosal injury. In: Malfertheiner P, Lundell L, Tytgat G. Update Gastroenterology: Novel developments in gastroenterology. EAGE 2006; 5-7.

3. Kikendall J. Pill induced oesophageal injury. Gastroenterol Clin North Am 1991; 20:835-846.

4. Jaspersen D. Drug-induced oesophageal disorders: pathogenesis, incidence, prevention and management. Drug Safety 2000; 22: 237-249.

5. Al-Mofarreh M, Al Mofleh I. Esophageal ulceration complicating doxycycline therapy. World J Gastroenterol 2003; 9: 609-611

6. Carlborg B, Densert O, Lindqvist C. Tetracycline induced esophageal ulcers. a clinical and experimental study. Laryngoscope 1983; 93:184. 
7. Dent J, Dodds W, Friedman R, Sekiguchi T, Hogan WJ, Arndorfer RC, et al. Mechanism of gastroesophageal reflux in recumbent asymptomatic human subjects. J Clin Invest 1980; 65:256.

8. Akbayir N, Alkim C, Erdem L, Sakiz D, Sokmen HM. A case report of doxycycline induced esophageal and gastric ulcer. Turk J Gastroenterol. 2002;13(4):232-235

9. Yap I, Guan R, Kang J, Gwee K, Tan C. Pillinduced esophageal ulcer. Singapore Med J. 1993; 34(3):257- 258.

10. Petersen K, Jaspersen D. Medication-induced oesophageal disorders. Expert Opin Drug Saf. 2003; 2(5):495- 507.

11. Baehr P, McDonald G. Esophageal disorders caused by infection, systemic illness, medications, radiation, and trauma. In: Feldman M, Scharschmidt BF, Sleisenger MH. Gastrointestinal and Liver Disease. $6^{\text {th }}$ edn. WB Saunders 1998: 519-39.

12. Hey H, Jorgensen F, Sorensen K, Hasselbalch H, Wamberg T. Oesophageal transit of six commonly used tablets and capsules. BMJ 1982; 285: 17171719.
13. Boyce HW. Drug-induced oesophageal damage: diseases of medical progress. Gastrointest Endosc 1998; 47: 547-550.

14. Carlborg B, Densert O. Oesophageal lesions caused by orally administered drugs. Eur Surg Res 1980; 12:270-282.

15. Beel RJ. Tetracycline induced oesophagitis. AI Med 1986; 50:47-50.

16. Applegate GR, Malmud LS, Rock E. It's a hard pill to swallow: or don't take it lying down (letter). Gastroenterology 1980; 78:1132.

Peer reviewer: Veysel Tahan, MD, University of Iowa Hospitals and Clinics, Department of Gastroenterology, Iowa City, IA, USA.

Mohamed I Radwan, Assistant professor of Tropical Medicine and Hepatogastroenterology, Faculty of Medicine, Zagazig University, Egypt.

Editor: Mohamed Emara, Lecturer of Tropical Medicine and Hepatogastroenterology, Faculty of Medicine, Zagazig University, Egypt. 